\title{
Eclosão e Mortalidade de Meloidogyne exigua em Extratos e em Produtos Naturais*
}

\author{
Sônia M. L. Salgado** \& Vicente P. Campos \\ Departamento de Fitopatologia, Universidade Federal de Lavras, Campus Universitário, Cx. Postal 37, CEP 37200-000, \\ Lavras, MG, Fax (035) 3829-1283, e-mail: solisa@ufla.br
}

(Aceito para publicação em 07/02/2003)

Autor para correspondência: Sônia M. Lima Salgado

SALGADO, S.M.L. \& CAMPOS, V.P. Eclosão e mortalidade de Meloidogyne exigua em extratos e em produtos naturais. Fitopatologia Brasileira 28:166-170. 2003.

\section{RESUMO}

A eclosão e mortalidade de juvenis de segundo estádio (J2) de Meloidogyne exigua foram avaliadas em extratos aquosos de urucum-colorau (Bixa orellana), cravo-da-índia (Syzygium aromaticum), canela (Cinnamomum zeylanicum), pimenta-do-reino (Piper nigrum), gengibre (Zingiber officinale), salsa (Petroselium crispum), soro de leite, solução nutritiva hidropônica, solução aquosa de cloreto de sódio $(\mathrm{NaCl})$ e açúcar (sacarose), fermento biológico e probiótico (Controlmix $\left.{ }^{\circledR}\right)$. O soro de leite e os extratos de canela, fermento biológico e cloreto de sódio causaram $100 \%$ de mortalidade $(\mathrm{P} \leq 0,05)$ após 24 h do contato dos $\mathrm{J} 2$ com os extratos. Mortalidade acima de 50\% também ocorreu nos extratos de cravo-da-índia e no probiótico. Os demais materiais testados foram estatisticamente iguais à água $(\mathrm{P} \leq 0,05)$. A eclosão foi avaliada durante 16 dias de imersão dos ovos de M. exigua nos extratos. Comparando os valores da área abaixo da curva de progresso da eclosão, observou-se que, exceto o açúcar e a solução hidropônica, todos os extratos e produtos testados inibiram a eclosão $(\mathrm{P} \leq 0,01)$ dos $\mathrm{J} 2$ de M. exigua. Entre todos os extratos e produtos testados, maior inibição $(\mathrm{P} \leq 0,01)$ da eclosão ocorreu no soro de leite, probiótico, canela e cravo-daíndia, destacando-se o soro de leite e o extrato de canela que assim como inibiram a eclosão também foram altamente tóxicos aos juvenis.

Palavras-chave adicionais: controle biológico, fitonematóides, extratos naturais, mortalidade.

ABSTRACT

Hatching and mortality of Meloidogyne exigua in extracts and in natural products

The hatching and mortality of Meloidogyne exigua secondstage juveniles (J2) were evaluated after $24 \mathrm{~h}$ in aqueous extracts of Bixa orellana, Cinnamomum zeylanicum, Petroselium crispum, Piper nigrum, Syzygium aromaticum, Zingiber officinale whey of milk, hidroponic solution, sodium chloride $(\mathrm{NaCl})$ and saccharose aqueous solution, yeast and probiotic (Controlmix ${ }^{\circledR}$ ) solutions. The whey of milk, C. zeylanicum, yeast and sodium chloride $(\mathrm{NaCl})$ solutions caused $100 \%$ mortality of $\mathrm{J} 2(\mathrm{P} \leq 0,05)$. Extracts of $S$.

aromaticum and Controlmix ${ }^{\circledR}$ probiotic caused more than $50 \%$ mortality of $\mathrm{J} 2(\mathrm{P} \leq 0,05)$. Hatching was evaluated by placing eggs of $M$. exigua in those different extracts and products for 16 days. The area under the curve of hatching progress, evaluated at $48 \mathrm{~h}$ intervals, showed that all extracts and products caused inhibition as compared to water $(\mathrm{P} \leq 0,01)$, except for saccharose and hidroponic solutions. Whey of milk, probiotic, C. zeylanicum and S. aromaticum accounted for greater inhibition, among all the tested extracts and products $(\mathrm{P} \leq 0,01)$. As shown, whey of milk and $C$. zeylanicum extract also caused $100 \%$ mortality of $\mathbf{J} 2$.

\section{INTRODUÇÃO}

Meloidogyne exigua Goeldi, 1887, é importante patógeno para a cafeicultura brasileira pois, além de estar bastante disseminado nos cafezais brasileiros, o efeito do parasitismo desse nematóide causou redução de 50 a 68,2\% nas produções iniciais do cafeeiro (Coffea arabica L.) (Guerra Neto et al., 1985). Meloidogyne exigua foi encontrado em $45,4 \%$ das amostras de solo e raízes coletadas em diversos cafezais de Minas Gerais (Souza et al., 1999) e em 26\% das amostras coletadas no Paraná (Portz et al., 2000).

Produtos naturais têm sido fonte de compostos para o

\footnotetext{
* Parte da Tese de Doutorado do primeiro autor. Universidade Federal de Lavras (2001).
}

controle de pragas e doenças (Jespers \& Waard, 1993; Isman, 2000). Substâncias com efeito nematicida têm sido encontradas em extratos obtidos de diversas espécies vegetais (Khurma \& Singii, 1997; Abid et al., 1997; Dias et al., 2000; Costa et al., 2001).

Produtos naturais, como os condimentos de origem vegetal, também conhecidos por especiarias, há muito são utilizados na alimentação humana com propósito de realçar o sabor e preservar os alimentos. Porém, só nas últimas décadas que estudos mais criteriosos têm sido realizados sobre suas propriedades antimicrobianas (Schmidt, 1994). Propriedades antimicrobianas têm sido encontradas no cravoda-índia [Syzygium aromaticum (L.) Merr. \& Perry] e canela (Cinnamomum zeylanicum Breyn) (Bullerman et al., 1977). Sangwan et al. (1990) observaram ação nematicida do óleo 
Eclosão e mortalidade de Meloidogyne exigua em extratos e em...

de cravo-da-índia a fitonematóides. Assim como a canela, o soro de leite, produzido e descartado na indústria queijeira, ainda não foi estudado quanto à presença de substâncias bioativas a fitonematóides.

Novos produtos de menor impacto ao homem e ao meio ambiente precisam ser estudados para o controle dos fitonematóides, incluindo extratos e produtos naturais, visando, futuramente, o controle populacional destes patógenos, o que poderá beneficiar principalmente a agricultura orgânica, na qual ocorre a proibição do uso de nematicidas oriundos da indústria petroquímica. Especificamente no caso de M. exigua, as pesquisas que utilizam produtos naturais no controle desse nematóide ainda são escassas. Objetivou-se, então, estudar o efeito in vitro de diversos extratos vegetais e produtos naturais na eclosão e mortalidade de $M$. exigua do cafeeiro.

\section{MATERIAL E MÉTODOS}

\section{Obtenção de ovos e juvenis de segundo estádio (J2) de $M$. exigua}

De raízes galhadas de cafeeiros infestados por $M$. exigua extraíram-se os ovos pela técnica de Hussey \& Barker (1973). Para facilitar a visualização desses ovos nos testes subseqüentes, a suspensão obtida foi submetida ao método de Flotação e Centrifugação proposto por Jenkins (1964). Os J2 de $M$. exigua foram obtidos após a incubação dos ovos em câmaras de eclosão preparadas empregando-se placas de Petri $(4,5 \mathrm{~cm})$ forradas com peneiras de tecido poliéster $(0,025 \mathrm{a}$ 0,030 $\mathrm{mm}$ de abertura) fixado em anéis de PVC (40 mm x 10 $\mathrm{mm}$ ). Os $\mathrm{J} 2$ eclodidos que atravessavam a peneira eram diariamente coletados.

\section{Preparo dos extratos}

De acordo com a disponibilidade e utilização para conservação de alimentos, foram selecionadas e adquiridas no comércio local as seguintes especiarias: botão floral de cravo-da-índia, casca de canela, pimenta-do-reino (Piper nigrum autoridade?) moída, urucum (Bixa orellana L.) na forma de colorau, todos comercializados com a marca Santa Bárbara ${ }^{\circledR}$, além de rizoma de gengibre (Zingiber officinale autoridade?), folhas frescas de salsa (Petroselinum crispum autoridade?). Além desses, empregaram-se, também, fermento biológico (Fleischmann®), cloreto de sódio (sal refinado iodado, marca Cisne ${ }^{\circledR}$ ), açúcar (sacarose), solução nutritiva para cultivo hidropônico de folhosas (Furlani, 1998), probiótico (Controlmix $\left.{ }^{\circledR}\right)$ e soro de leite. Pela infusão em água, prepararam-se os extratos das especiarias bem como as soluções de cloreto de sódio e sacarose, todos na concentração de $5 \%(\mathrm{p} / \mathrm{v})$. O fermento biológico e o probiótico (Controlmix®) foram colocados em água à temperatura ambiente. Após $15 \mathrm{~h}$, realizou-se a filtração em papel Whatman $\mathrm{n}^{\circ} 1$ colocado em funil Buchner. O soro de leite in natura e a solução hidropônica foram empregados na constituição original. Todos os extratos e demais produtos foram armazenados em freezer e, descongelados por $4 \mathrm{~h}$ à temperatura ambiente antes da utilização.

\section{Avaliação da eclosão de $\mathbf{J} 2$ de $M$. exigua nos extratos e produtos naturais}

Em câmaras de eclosão, preparadas como descrito anteriormente, colocaram-se 700 ovos de M. exigua em $1 \mathrm{ml}$ de água e $5 \mathrm{ml}$ do extrato de cravo da índia, canela, pimentado-reino, urucum, gengibre, salsa, ou das soluções: hidropônica, cloreto de sódio ou açúcar, ou dos filtrados de fermento biológico e probiótico (Controlmix $®$ ) ou do soro de leite. As câmaras de eclosão foram mantidas em temperatura ambiente. $\mathrm{O}$ ensaio foi montado em delineamento inteiramente casualizado com 13 tratamentos e quatro repetições, empregando-se água como testemunha. A contagem dos $\mathbf{J} 2$ eclodidos iniciou-se $48 \mathrm{~h}$ após a montagem do ensaio e prosseguiu nos 16 dias subseqüentes de incubação, com intervalos de $48 \mathrm{~h}$. Conforme o material-teste era retirado da câmara de eclosão para avaliação dos $\mathrm{J} 2$ eclodidos, novo volume $(5 \mathrm{ml})$ do mesmo material (extrato ou produto natural) foi colocado na câmara de eclosão para a próxima avaliação. A análise da eclosão dos $\mathrm{J} 2$ de $M$. exigua foi feita por meio da área abaixo da curva de progresso, calculada pela equação proposta por Campbell \& Madden (1990):

$$
\mathrm{AACPE}=\sum_{\mathrm{i}=1}^{\mathrm{n}-1} \frac{\mathrm{Y}_{\mathrm{i}}+\mathrm{Y}_{\mathrm{i}+1}}{2} *\left(\mathrm{~T}_{\mathrm{i}-1}-\mathrm{T}_{\mathrm{i}}\right)
$$

Os valores da porcentagem de $\mathrm{J} 2$ eclodidos foram aplicados na fórmula considerando: $\mathrm{Y}_{\mathrm{i}}=$ porcentagem de eclosão na i-ésima avaliação; $\mathrm{T}_{\mathrm{i}}=$ tempo em dias na i-ésima avaliação; $n$ = número de avaliações. Desse modo obteve-se a AACPE = área abaixo da curva de progresso da eclosão. Os dados da AACPE foram analisados pelo programa Sisvar® versão 4.3 (Build 42), mediante transformação para $\log$ (x). As diferenças estatísticas foram detectadas pelo teste Scott \& Knott (1974) a 1\% de significância.

\section{Mortalidade de $\mathbf{J} 2$ de $M$. exigua nos extratos e produtos naturais}

Em lâminas escavadas (150 ml) colocaram-se $120 \mathrm{ml}$ do extrato ou produto-teste e $30 \mathrm{ml}$ da suspensão de $\mathrm{J} 2$, com 25 espécimes eclodidos nas últimas $24 \mathrm{~h}$. As lâminas foram mantidas em câmara úmida preparada em placa de Petri $(90$ $\mathrm{mm}$ ) com papel de filtro umedecido. $\mathrm{O}$ ensaio foi montado em delineamento inteiramente casualizado, empregando-se os mesmos extratos e soluções usados no ensaio de eclosão (13 tratamentos e quatro repetições). A avaliação da mortalidade foi realizada com $24 \mathrm{~h}$ utilizando-se metodologia proposta por Chen \& Dickson (2000). Calculou-se a porcentagem de $\mathrm{J} 2$ mortos do total de $\mathrm{J} 2$ observados em cada parcela. Os dados referentes à porcentagem de $\mathrm{J} 2$ mortos foram analisados pelo programa estatístico Genmod-SAS, e as diferenças estatísticas foram detectadas pelo teste Qui-Quadrado a $5 \%$ de significância.

\section{RESULTADOS E DISCUSSÃO}

Maior mortalidade de J2 de M. exigua $(\mathrm{P} \leq 0,05)$ ocorreu no soro de leite, canela, fermento biológico e na 
solução de cloreto de sódio (Tabela 1). A alta mortalidade dos J2 no cloreto de sódio, pode ter sido causada pela concentração de íons $\mathrm{Na}^{+}$e $\mathrm{Cl}^{-}$, que provavelmente provocaram alteração na regulação osmótica do nematóide. Variação na concentração de íons na solução de $\mathrm{NaCl}$ alterou a capacidade de regulação osmótica de Panagrellus redivivus (Linne, 1767) Goodey, 1945 incubados por 25 h nessa solução (Krusberg, 1971).

A alta mortalidade de $M$. exigua no soro de leite, fermento biológico e probiótico provavelmente ocorreu pela variação na taxa de $\mathrm{O}_{2}$ e $\mathrm{CO}_{2}$, resultante da ativação do metabolismo dos microrganismos constituintes destes materiais, na presença de água. Bactérias, fungos e leveduras são encontradas no soro de leite (Viotto, 1993), e provavelmente modificaram a concentração de dióxido de carbono, reduzindo o oxigênio disponível que, segundo Rohde (1971), é essencial para as atividades metabólicas normais dos nematóides. Este mesmo efeito sobre os $\mathrm{J} 2$ pode ter ocorrido no fermento biológico, constituído de Saccharomyces cerevisiae Meyen ex Hansen, (Muchovej \& Muchovej, 1989), e no probiótico, constituído por diversas bactérias. Além disso, existe a possibilidade de acúmulo de substâncias bioativas resultantes do metabolismo destes microrganismos. De fato, S. cerevisae produz substâncias com ação fungicida comprovada (Stangarlin \& Pascholati, 1994). No entanto, a presença de substâncias bioativas tóxicas a fitonematóides em culturas de $S$. cerevisae ainda não tem sido pesquisada, assim como aquelas substâncias produzidas pelas bactérias do probiótico.

Das especiarias testadas, maior efeito tóxico a $M$. exigua foi demonstrado pelo extrato de canela, onde $100 \%$ dos J2 morreram. Entretanto, nesse extrato ainda não se conhece o princípio ativo capaz de matar os $\mathrm{J} 2$ de $M$. exigua, necessitando, portanto, de pesquisas para a determinação da causa da alta toxicidade desse material sobre $M$. exigua.

O cravo-da-índia causou mais de $60 \%$ de mortalidade de J2 (Tabela 1). O composto eugenol, presente no cravo-daíndia (Bullerman et al., 1977), foi possivelmente responsável pela alteração nos sistemas vitais dos J2. De fato, o eugenol causou alta mortalidade de $\mathrm{J} 2$ de $M$. incognita (Kofoid \& White) Chitwood (Chatterjee et al., 1982), Anguina tritici (Steinbuch, 1799) Chitwood, 1935, Tylenchulus semipenetrans Cobb, M. javanica (Treub) Chitwood e $H$. cajani Koshi, 1967 (Sangwan et al., 1990).

Apesar de diferir $(\mathrm{P} \leq 0,05)$ da testemunha (água), o extrato de pimenta-do-reino causou menos de $12 \%$ de mortalidade de $\mathbf{J} 2$ (Tabela 1), demonstrando conter substâncias talvez menos tóxicas aos $\mathrm{J} 2$ de $M$. exigua ou em menor concentração.

Sabe-se que ovos com células e embriões em diversos estádios de desenvolvimento são comumente encontrados nas massas de ovos e, possivelmente, na suspensão de ovos extraídos dessas massas, causando grande variação na eclosão ao longo do tempo. Diante disso, a integralização da eclosão dos J2 ao longo do período de incubação dos ovos, através da AACPE, pode representar melhor os resultados. Pela análise
TABELA 1- Mortalidade e área abaixo da curva de progresso da eclosão (AACPE) de juvenis de segundo estádio (J2) de Meloidogyne exigua em extratos de especiarias aromáticas, probiótico (Controlmix $\left.{ }^{\circledR}\right)$, solução nutritiva de cultivo hidropônico de folhosas e demais produtos naturais

\begin{tabular}{lcc}
\hline \hline Tratamento & $\begin{array}{c}\text { Mortalidade* } \\
(\%)\end{array}$ & AACPE* \\
\hline Soro de leite & $100,0 \mathrm{a}$ & $13,3 \mathrm{a}$ \\
Canela (Cinnamomum zeylanicum ) & $100,0 \mathrm{a}$ & $35,82 \mathrm{~b}$ \\
Fermento biológico & $100,0 \mathrm{a}$ & $209,90 \mathrm{~d}$ \\
Cloreto de sódio (NaCl) & $100,0 \mathrm{a}$ & $67,45 \mathrm{c}$ \\
Cravo-da-índia (Syzygium aromaticum $)$ & $68,8 \mathrm{~b}$ & $45,52 \mathrm{~b}$ \\
Probiótico (Controlmix@) & $50,7 \mathrm{~b}$ & $28,8 \mathrm{~b}$ \\
Pimenta-do-reino (Pipper nigrum) & $11,8 \mathrm{c}$ & $274,67 \mathrm{~d}$ \\
Açúcar (Sacarose) & $4,6 \mathrm{c} \mathrm{d}$ & $737,15 \mathrm{e}$ \\
Salsa (Petroselinum crispum) & $3,1 \mathrm{c} \mathrm{d}$ & $230,92 \mathrm{~d}$ \\
Gengibre (Zingiber officinale ) & $1,0 \mathrm{~d}$ & $107,32 \mathrm{c}$ \\
Urucum-colorau (Bixa orellana) & $0,0 \mathrm{~d}$ & $433,42 \mathrm{~d}$ \\
Solução hidropônica & $0,0 \mathrm{~d}$ & $631,60 \mathrm{e}$ \\
Água & $0,0 \mathrm{~d}$ & $769,50 \mathrm{e}$ \\
\hline
\end{tabular}

*Valores da mortalidade e da AACPE seguidos da mesma letra são iguais, respectivamente, pelo teste Qui-Quadrado - SAS $(\mathrm{P} \leq 0,05)$ e Scott-Knott $(\mathrm{P} \leq 0,01)$

da AACPE, o soro de leite causou maior inibição da eclosão de $M$. exigua $(\mathrm{P} \leq 0,01)$. Esse efeito tóxico foi observado também sobre os juvenis, onde $100 \%$ dos $\mathrm{J} 2$ foram mortos (Tabela 1). Assim como o soro de leite, o probiótico, canela e cravo-da-índia reduziram drasticamente a eclosão dos J2, demonstrada pelos menores valores da AACPE (Tabela 1).

Todos os tratamentos que causaram 50 a $100 \%$ de mortalidade de $\mathrm{J} 2$ de $M$. exigua também apresentaram menores valores de AACPE, ou seja, causaram redução $(\mathrm{P} \leq 0,01)$ na eclosão (Tabela 1). Khurma \& Singii (1997), encontraram efeito tanto na mortalidade quanto na eclosão de $\mathrm{J} 2$ de $M$. incognita e $M$. javanica, quando usaram extrato de sementes de Sesbania sesban (L.) Merr. Por outro lado, o extrato de gengibre, embora não tenha causado mortalidade de $\mathrm{J} 2$, inibiu a eclosão $(\mathrm{P} \leq 0,01)$, assim como o extrato de urucum-colorau. Dentre esses dois extratos, o gengibre foi mais eficiente em reduzir a eclosão, com menor valor da AACPE do que o colorau (Tabela 1).

Verifica-se que as maiores eclosões ocorreram igualmente $(\mathrm{P} \leq 0,01)$ em água, solução nutritiva e açúcar (Tabela 1), com maiores valores de AACPE.

O progresso da eclosão dos juvenis de $M$. exigua em água, comparado com soro de leite, canela, cravo-da-índia e probiótico nos 16 dias de incubação dos ovos (Figura $1 \mathrm{~A}, \mathrm{~B}$, C e D), demonstrou que o efeito tóxico ocorre já nos primeiros dias. Nos extratos de canela e cravo-da-índia, pequena eclosão ocorreu nos primeiros seis dias, a qual foi totalmente paralisada a partir de então (Figura 1 B e C). Essa pequena eclosão nos primeiros dias provavelmente ocorreu pela saída do ovo de alguns dos juvenis já formados, e a posterior paralisação da eclosão nesses extratos ocorreu porque a multiplicação celular e as fases do desenvolvimento embrionário foram afetadas na mesma intensidade. Situação semelhante verificou-se 
Eclosão e mortalidade de Meloidogyne exigua em extratos e em...
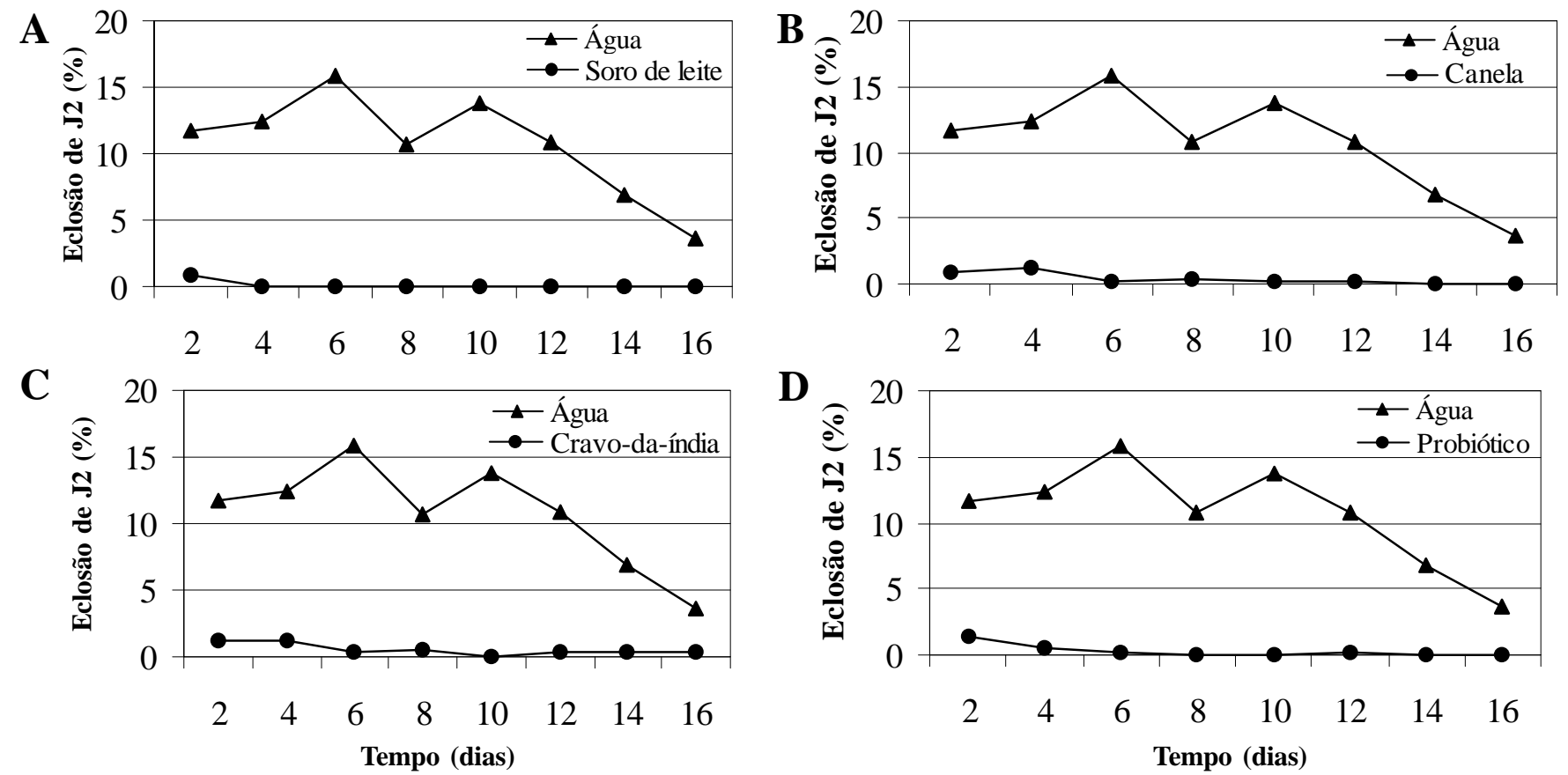

FIG. 1 - Curvas de progresso da eclosão de juvenis do segundo estádio (J2) de Meloidogyne exigua em água comparado com alguns dos materiais de alta toxicidade aos J2. A) Soro de leite; B) canela (Cinnamomum zeylanicum); C) cravo-da-índia (Syzygium aromaticum) e D) Probiótico (Controlmix ${ }^{\circledR}$ ). Valores da eclosão são dados originais da porcentagem média de J2 eclodidos das quatro repetições em cada avaliação (tempo em dias).

no probiótico (Controlmix $\left.{ }^{\circledR}\right)$, onde a eclosão de $\mathrm{J} 2$ de $M$. exigua ocorreu somente nos primeiros quatro dias de incubação dos ovos que, possivelmente, já continham o juvenil desenvolvido. A partir dos quatro dias, ocorreu uma redução na eclosão que foi praticamente inibida a partir do $6^{\circ}$ dia de incubação, o que possivelmente ocorreu pelo efeito tóxico do probiótico ter sido mais prejudicial ao desenvolvimento embrionário do que no juvenil já formado (Figura $1 \mathrm{D})$. No soro de leite ocorreu paralisação da eclosão desde o início até o final do ensaio, demonstrando que o efeito tóxico foi igual tanto no juvenil formado como durante o desenvolvimento embrionário. Em água a eclosão aumentou entre o quarto e o sexto dia de incubação dos ovos, seguido de novo aumento e queda progressiva a partir do décimo dia (Figura 1).

A solução hidropônica e o açúcar (sacarose) não apresentaram efeito tóxico a $M$. exigua. Isso pode ser observado pela ausência de $\mathrm{J} 2$ mortos e pela alta eclosão, indicada pelos valores de AACPE (Tabela 1), estatisticamente iguais à água $(\mathrm{P} \leq 0,01)$. Porém todos os demais materiais testados apresentaram algum efeito tóxico a $M$. exigua. A maioria dos extratos e demais produtos foi mais expressivo na inibição da eclosão de $\mathrm{J} 2$ de $M$. exigua. Isso sugere que tais extratos contêm substâncias diferentes ativamente envolvidas na mortalidade e na inibição da eclosão, ou ainda que, as diferentes fases envolvidas no processo de eclosão, como a multiplicação celular, o desenvolvimento embrionário, troca de cutícula e saída do ovo (Campos et al., 2001) podem estar sendo diferentemente afetados pelas substâncias e suas concentrações nos extratos.
Nas concentrações utilizadas, o soro de leite, fermento biológico, canela e cloreto de sódio, foram tóxicos a J2 de $M$. exigua, enquanto que o probiótico, cravo-da-índia, canela e soro de leite foram eficazes na redução da eclosão. A solução nutritiva, comumente empregada no cultivo hidropônico de folhosas, permite a eclosão e sobrevivência de $\mathrm{J} 2$ de $M$. exigua, necessitando, por conseguinte, de mais estudos, inclusive com outros fitonematóides.

\section{REFERÊNCIAS BIBLIOGRÁFICAS}

ABID, M., CHOUDHARY, M.I., MAQBOOL, M.A. \& RAHMAN, A.U. Preliminary screening of some plants for their nematicidal activity against Meloidogyne javanica. Nematologia Mediterranea 25:155-157. 1997.

BULLERMAN, L.B., LIEU, F.Y. \& SEIER, S.A. Inhibition of growth and aflatoxin production by cinnamon and clove oils, cinnamic aldehyde and eugenol. Journal of Food Science 42:1107-1109. 1977.

CAMPBELL, C.L. \& MADDEN, L.V. Introduction to plant disease epidemiology. New York: John Wiley \& Sons, 1990.

CAMPOS, V.P., CAMPOS, J.R., SILVA, L.H.C.P. \& DUTRA, M.R. Manejo de nematóides em hortaliças. In: Silva, L.H.C.P., Campos, J.R. \& Nojosa, G.B.A. (Eds.) Manejo integrado de doenças e pragas em hortaliças. Lavras: UFLA. 2001. pp. 125-158.

CHATTERJEE, A., SUKUL, N.C., LASKAR, S., \& GHOSHMAJUMDAR, S. Nematicidal principles from two species of lamiaceae. Journal of Nematology 14:118-120. 1982.

CHEN, S.Y. \& DICKSON, D.W. A technique for determining live second-stage juveniles of Heterodera glycines. Journal of Nematology 32:117-121. 2000. 
COSTA, M.J.N., CAMPOS, V.P., OLIVEIRA, D.F. \& PFENNING, L.H. Toxicidade de extratos vegetais e de estercos a Meloidogyne incognita. Summa Phytopathologica 27:245-250. 2001.

DIAS, C.R., SCHWAN, A.V., EZEQUIEL, D.P., SARMENTO, M.C. \& FERRAZ, S. Efeito de extratos aquosos de plantas medicinais na sobrevivência de juvenis de Meloidogyne incognita. Nematologia Brasileira 24:203-210. 2000.

FURLANI, P.R. Instruções para o cultivo de hortaliças de folhas pela técnica de hidroponia - NFT. Campinas. Instituto Agronômico. Boletim Técnico, 168. 1998.

GUERRA NETO, E.G., D'ANTONIO, A.M. \& FREIRE, A.C.F. Influência de Meloidogyne exigua Goeldi, 1887, no desenvolvimento de lavoura de Coffea arabica L., variedade Mundo Novo. Resumos, $12^{\circ}$ Congresso Brasileiro de Pesquisas Cafeeiras, Caxambu, MG. 1985. pp.36-37.

HUSSEY, R.S. \& BARKER, R.K. A comparison of methods of collecting inocula of Meloidogyne spp., including a new technique. Plant Disease Reporter 57:1025-1028. 1973.

ISMAN, M.B. Plant essential oils for pest and disease management. Crop Protection 19:603-608. 2000.

JENKINS, W.R. A rapid centrifugal-flotation technique for separating nematodes from soil. Plant Disease Reporter 48:692. 1964.

JESPERS, A.B.K. \& WAARD, M.A. de. Natural products in plant protection. Netherlands Journal Plant Pathology 99:109-117. 1993

KHURMA, U.R. \& SINGII, A. Nematicidal potential of seed extracts: in vitro effects on juvenile mortality and egg hatch of Meloidogyne incognita and M. javanica. Nematologia Mediterranea 25:49-54. 1997.

KRUSBERG, L.R. Chemical composition of nematodes. In: Zuckerman, B.M., Mai, W.F. \& Rohde, R.A. (Eds.) Plant parasitic nematodes. II. Cytogenetics, host parasite interactions and physiology, New York. Academic Press. 1971. pp.213-234.

MUCHOVEJ, J.J. \& MUCHOVEJ, R.M.C. Noções básicas de Micologia. Viçosa. UFV. 1989.

PORTZ, R.L., FURLANETTO, C. \& STANGARLIN, J.R. Levantamento de espécies de nematóides do gênero Meloidogyne na cultura do café em municípios do oeste do Paraná. Fitopatologia Brasileira 25:339. 2000.

ROHDE, R.A. Respiration. In: Zuckerman, B.M., Mai, W.F. \& Rohde, R.A. (Eds.) Plant parasitic nematodes. II. Cytogenetics, host parasite interactions and physiology. New York: Academic Press. 1971. pp.235-246.

SANGWAN, N.K., VERMA, B.S., VERMA, K.K. \& DHINDSA, K.S. Nematicidal activity of some essential plant oils. Pesticide Science 28:331-335. 1990.

SCHMIDT, L.F. Efeito de extratos naturais de origem vegetal sobre esporos de Desulfotomaculum nigrificans. (Tese de Doutorado), Campinas. Universidade de Campinas. 1994.

SCOTT, A.J. \& KNOTT, M. Cluster analysis method for grouping means in the analysis of variance. Biometrics 30:507-512. 1974.

SOUZA, J.T., MAXIMINIANO, C. \& CAMPOS, V.P. Nematóides parasitos encontrados em cafeeiros em campo e em viveiros de mudas do Estado de Minas Gerais. Summa Phytopathologica 25:180-183. 1999.

STANGARLIN, J.R. \& PASCHOLATI, S.F. Proteção de plântulas de milho pipoca contra Exserohilum turcicum pelo uso de Saccaromyces cerevisiae. Summa Phytopathologica 20:16-21. 1994.

VIOTTO, W.H. Ultrafiltração de soro doce de queijo minas frescal. Efeito de pré-tratamentos do soro no desempenho da membrana e na composição e solubilidade do concentrado protéico de soro. (Tese de Doutorado), Campinas.Universidade de Campinas. 1993. 